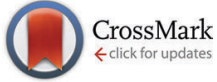

Cite this: Phys. Chem. Chem. Phys., 2016, 18, 27733

Received 15th August 2016, Accepted 26th September 2016

DOI: $10.1039 / c 6 c p 05658 j$

www.rsc.org/pccp

\section{A redox-active radical as an effective nanoelectronic component: stability and electrochemical tunnelling spectroscopy in ionic liquids $\dagger$}

\author{
Alexander V. Rudnev, ${ }^{\mathrm{ab}}$ Carlos Franco, ${ }^{\mathrm{c}}$ Núria Crivillers, ${ }^{* \mathrm{c}}$ Gonca Seber, ${ }^{\mathrm{c}}$ \\ Andrea Droghetti, ${ }^{d}$ Ivan Rungger, ${ }^{e}$ Ilya V. Pobelov, ${ }^{a}$ Jaume Veciana, ${ }^{c}$ \\ Marta Mas-Torrent ${ }^{* c}$ and Concepció Rovira*c
}

\begin{abstract}
A redox-active persistent perchlorotriphenylmethyl (PTM) radical chemically linked to gold exhibits stable electrochemical activity in ionic liquids. Electrochemical tunnelling spectroscopy in this medium demonstrates that the PTM radical shows a highly effective redoxmediated current enhancement, demonstrating its applicability as an active nanometer-scale electronic component.
\end{abstract}

Redox-active molecules have been shown to be promising active components for developing nanometer-scale electronic devices ${ }^{1-3}$ capable of acting as current rectifiers ${ }^{4}$ and switches,${ }^{5-7}$ mechanical switches ${ }^{8,9}$ or active components in charge-storage devices. ${ }^{10}$ In order to progress in this field, the control of the molecular redox state as well as the understanding of its influence on the charge transport properties is crucial. Experiments on electrochemicallygated charge transport across redox-active molecular junctions have demonstrated an effective current modulation. ${ }^{11,12}$ In these systems, the Marcus-type reorganization energy strongly influences the efficiency of the current enhancement due to redox mediated electron tunnelling.

Organic radical perchlorotriphenylmethyl (PTM) is persistent and stable, both chemically and thermally, due to the steric hindrance at the central carbon atom caused by the orthochlorine atoms of the phenyl rings (Fig. 1a). ${ }^{13}$ Importantly, PTM radicals display a low reduction potential to the corresponding PTM anions, ${ }^{14}$ which makes them very interesting molecular

\footnotetext{
${ }^{a}$ University of Bern, Department of Chemistry and Biochemistry, Freiestrasse 3, 3012 Bern, Switzerland. E-mail: rudnev@dcb.unibe.ch

${ }^{b}$ Russian Academy of Sciences A.N. Frumkin Institute of Physical Chemistry and Electrochemistry RAS, Leninskii pr. 31, Moscow, 119991, Russia

${ }^{c}$ Department of Molecular Nanoscience and Organic Materials, Institut de Ciència de Materials de Barcelona (ICMAB-CSIC) and CIBER-BBN, Campus la Universitat Autonoma Barcelona (UAB), 08193 Bellaterra, Spain. E-mail: ncrivillers@icmab.es, mmas@icmab.es, cun@icmab.es

${ }^{d}$ Nano-Bio Spectroscopy Group and European Theoretical Spectroscopy Facility (ETSF), Universidad del Pais Vasco CFM, CSIC-UPV/EHU-MPC \& DIPC,

Avenida Tolosa 72, 20018 San Sebastian, Spain

${ }^{e}$ Materials Division, National Physical Laboratory, Teddington, TW11 OLW, UK $\dagger$ Electronic supplementary information (ESI) available: Experimental details, additional results and figures. See DOI: $10.1039 / \mathrm{c} 6 \mathrm{cp} 05658 \mathrm{j}$
}

(a)
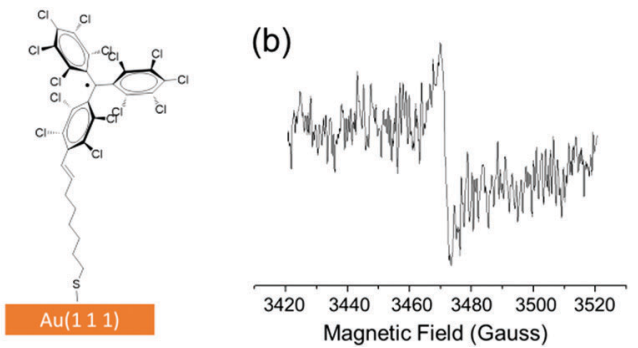

Fig. 1 (a) Schematics of PTM-C8-SH adsorbed on Au(111) and (b) EPR spectrum of PTM-C8-SH SAM on Au(111) recorded at $300 \mathrm{~K}$ under ambient conditions.

redox switches. ${ }^{15-17}$ The charge transport through self-assembled monolayers $^{18,19}$ and single-molecule junctions ${ }^{20}$ incorporating PTM radicals have been studied before, but the different redox states (radical and anion) were not addressed. Since the structure of both components of the redox pair is almost identical, ${ }^{21}$ the inner reorganization energy associated with the structural change upon reduction/oxidation is expected to be low. In addition, bulky redox systems are expected to show low outer-sphere reorganization energy associated with the surrounding solvent, and indeed their current enhancement efficiency in redox-active molecular junctions is typically high. ${ }^{22-24}$ In view of this, the bulky organic radical perchlorotriphenylmethyl (PTM) is expected to be a very good redox mediator.

Here, the electrochemical gating of the charge transport through a surface-bound PTM radical has been explored using an electrochemical scanning tunnelling spectroscopy (EC-STS) technique. We demonstrated current enhancement due to the redox-mediated electron tunnelling (RMET) mechanism ${ }^{23-41}$ involving the PTM radical as the active redox-centre. Our results show that, in comparison with other redox-active moieties, the PTM radical is among the most efficient redox mediators in RMET, demonstrating its applicability as an active electronic component in electronic devices of nanoscale size.

A PTM radical bearing an alkenyl chain spacer with a thiol anchoring group (PTM-C8-SH) was synthesised as described 
previously $^{18}$ and used to prepare self-assembled monolayers (SAMs) on $\mathrm{Au}(111)$ (Fig. 1a). Fig. 1a shows the schematics of PTM-C8-SH assembled on Au(111) (see the ESI $\dagger$ for the characterization and assembly details). The Electron Paramagnetic Resonance spectrum of the SAM on $\mathrm{Au}(111)$ recorded at $300 \mathrm{~K}$ exhibited a signal with a $g$ value of 2.0032 and a line width of 2.6 Gauss (Fig. 1b), which shows that the PTM radical character is preserved once the molecule is immobilized on the gold surface. For the purpose of comparison, the electrochemically inactive $\alpha$ H-PTM-C8-SH (ESI, $\dagger$ Scheme S1) was isolated during the synthesis of the radical derivative and used to perform reference voltammetric and STS measurements.

Cyclic voltammograms (CVs) of PTM-C8-SH SAM on Au(111) in acetonitrile solution (ESI, $\dagger$ Fig. S1) showed the reduction and oxidation peaks assigned to the reversible reduction of the PTM radical moieties $\left(\mathrm{PTM}^{\bullet} \rightleftharpoons \mathrm{PTM}^{-}\right)$. The redox response was stable during a repetitive potential cycling and the linear dependence of the peak heights on the potential scan rate indicates the oxidation/reduction of a surface-confined species (ESI, $\dagger$ Fig. S1). However, organic solvents are not suitable for electrochemical STM/STS experiments due to their high volatility and/or insufficient stability of coatings employed to electrochemically insulate the STM tip. The PTM anion can be readily protonated in an acidic aqueous medium. Hence ionic liquids (ILs) appear to be the best media for EC-STM/STS measurements in our case. ${ }^{11,22}$

According to previous works, drying ILs by using molecular sieves ( $3 \AA$ ) may significantly decrease the water content. ${ }^{42,43}$ For this, hot molecular sieves activated at $200{ }^{\circ} \mathrm{C}$ were used to dry ILs before each experiment and they were also put into the electrochemical cell. The CVs of PTM-C8-SH on Au(111) were acquired using different ILs as electrolytes (ESI, $\dagger$ Table S1). A decrease of the redox signal with time (Fig. 2a) was observed and after 20 cycles (20 $\mathrm{min}$ ) the $\mathrm{CV}$ response indicated that the radical SAM became redox-inactive (dashed curve). However, the capacitance current did not change and remained much smaller than for the bare $\mathrm{Au}(111)$ (ESI, $\dagger$ Fig. S2a), demonstrating that the desorption of the monolayer does not take place. Fig. S2b (ESI $\dagger)$ shows a representative EC-STM image of PTM-C8-SH on $\mathrm{Au}(111)$ in $[\mathrm{Emim}]\left[\mathrm{EtSO}_{4}\right]$ recorded after the disappearance of the redox peaks. As illustrated by a cross-section
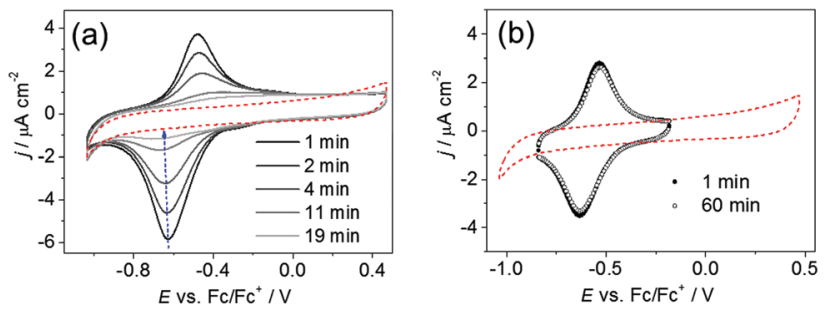

Fig. 2 (a and b) CVs of PTM-C8-SH on Au(111): (a) in $\left[\mathrm{Bmim}^{-}\left[\mathrm{BF}_{4}\right]\right.$ and (b) in $[\mathrm{Bmim}]\left[\mathrm{BF}_{4}\right]+\mathrm{Et}_{3} \mathrm{~N}(15: 1 \mathrm{v} / \mathrm{v})$ with molecular sieves at the $2 \mathrm{nd}$ and 60 th minutes from the beginning of potential cycling. Dashed curves are the CVs of the redox-inactive $\alpha \mathrm{H}$-PTM-C8-SH on Au(111). The scan rate was $50 \mathrm{mV} \mathrm{s}^{-1}$. The time from the beginning of continuous potential cycling is indicated.
(Fig. S2c, ESI $\dagger$ ), the STM image shows a rather uniform corrugation height of $\sim 0.8 \mathrm{~nm}$, which confirms unambiguously the presence of the adlayer. The above-mentioned results are in accordance with the well-known acidic character of the imidazolium cation ${ }^{44}$ (see $\mathrm{ESI} \dagger$ for a more detailed explanation), which could promote the protonation of the electrochemically generated anion forming the non-electroactive $\alpha$ H-PTM-C8-SH SAM on Au(111) (ESI, $\dagger$ Scheme S1). In order to corroborate it, a solution of the non-functionalized PTM anion $\left(\mathrm{PTM}^{-} \mathrm{K}^{+}[18-\mathrm{Crown}-6]\right)^{45}$ in $[$ Emim $]\left[\mathrm{EtSO}_{4}\right]$ was monitored using UV-Vis spectroscopy. The characteristic band of the $\mathrm{PTM}^{-}$at $500 \mathrm{~nm}$ was observed to decrease gradually with time (ESI, $\dagger$ Fig. S3a), and completely disappeared after $24 \mathrm{~h}$, confirming the protonation of the PTM anion in the IL, even after exhaustive drying. The same CV experiment was performed with the PTM SAM using $\left[\mathrm{SEt}_{3}\right]\left[\mathrm{Tf}_{2} \mathrm{~N}\right]$ in which, although a higher stability of the layer was observed, the redox peaks also diminished with time. We believe that small traces of water in the ionic medium could also induce the protonation of the PTM anion.

In order to overcome this problem, a soft base like triethylamine $\left(\mathrm{Et}_{3} \mathrm{~N}\right)$ was added to the IL $(1: 15 \mathrm{v} / \mathrm{v})$. According to the voltammetric and UV-Vis measurements, its presence greatly improved the stability of the PTM anion in the IL media. The CVs of PTM-C8-SH on Au(111) did not change significantly after $1 \mathrm{~h}$ of potential cycling (Fig. 2b) and the absorption band of $\mathrm{PTM}^{-}$remained unaltered after 24 hours (ESI, $\dagger$ Fig. S3b). We notice that the redox peaks of PTM-C8-SH on $\mathrm{Au}(111)$ in the ILs have a larger peak-to-peak separation as compared to those in acetonitrile (Fig. S1, ESI $\dagger$ ). We attribute such a behaviour to the slow ion pairing of the PTM anion with the cations of the ILs. ${ }^{46}$ Moreover, peak-to-peak separation differs for different ionic liquids, also suggesting that the shape of the voltammograms is affected by the IL-cation and PTM anion interactions (Fig. 2 and Fig. S4, S5, ESI $\dagger$ ).

Fig. 3 shows the CV of PTM-C8-SH SAM on Au(111) in $\left[\mathrm{SEt}_{3}\right]\left[\mathrm{Tf}_{2} \mathrm{~N}\right]+\mathrm{Et}_{3} \mathrm{~N}(15: 1 \mathrm{v} / \mathrm{v})$ recorded in the STM cell (Fig. 3a) and a representative EC-STM image (Fig. 3b). The redox response was stable and nearly identical to that obtained in the standard glass electrochemical cell. As expected, a highly ordered monolayer was not observed due to the bulkiness of the PTM moiety, which hinders the formation of a well-arranged molecular layer induced by the lateral interactions between the alkyl chains. However, the STM image demonstrates the formation of a
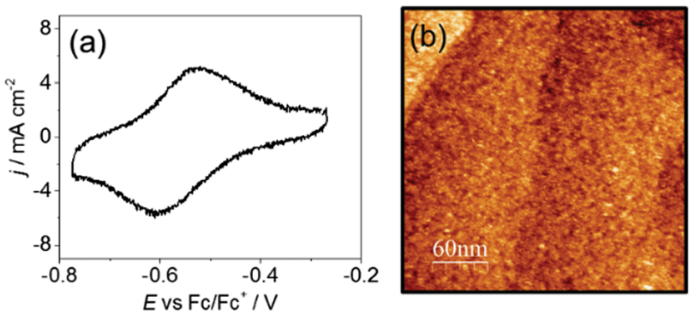

Fig. 3 (a) CV of PTM-C8-SH on Au(111) in $\left[\mathrm{Se}_{3}\right]\left[\mathrm{Tf}_{2} \mathrm{~N}\right]+\mathrm{Et}_{3} \mathrm{~N}(15: 1 \mathrm{v} / \mathrm{v})$ with molecular sieves as recorded in an STM cell and (b) EC-STM image recorded at $E_{\mathrm{S}}=-0.33 \mathrm{~V}, E_{\mathrm{T}}=-0.03 \mathrm{~V}$, and $I_{\text {SetPoint }}=40 \mathrm{pA}$. 
compact adlayer (see also Fig. S6 in the ESI $\dagger$ ). The coverage estimated from the integration of the $\mathrm{CV}$ peaks (with background current correction) was $1.2 \times 10^{-10} \mathrm{~mol} \mathrm{~cm}^{-2}$, which is $\approx 70 \%$ of the theoretical maximum coverage calculated from the area of a single PTM-C8-S-Au (see the ESI $\dagger$ for details).

After the radical monolayer was assembled on $\mathrm{Au}(111)$ and confirmed to be stable, electrochemical STS measurements in the constant bias mode were performed. ${ }^{23}$ In this mode, the potentials of both working electrodes, i.e. substrate $E_{\mathrm{S}}$ and tip $E_{\mathrm{T}}$, were swept simultaneously while keeping the bias voltage $E_{\text {bias }}=E_{\mathrm{T}}-E_{\mathrm{S}}$ constant. The STS curves $\left(I_{\mathrm{t}} v s . E_{\mathrm{S}}\right)$ were recorded at a fixed geometry of the tunnelling junction with the feedback being temporarily switched off during the acquisition of each STS curve. The results obtained for PTM-C8-SH on Au(111) are shown in Fig. 4a, in which a significant enhancement of the tunnelling current flowing between the substrate and the tip is observed. As expected, the control STS experiment with the redox-inactive $\alpha \mathrm{H}$-PTM-C8-SH monolayer showed a constant current without tunnelling enhancement (Fig. 4b).

Fig. 4c shows the master curve obtained from the histogram where each data point corresponds to the value of tunnelling current measured with the highest probability at a given potential value. The curve has a bell shape with a maximum located close to the equilibrium potential of the $\mathrm{PTM}^{\bullet} / \mathrm{PTM}^{-}$ redox pair ( $c f$. the $\mathrm{CV}$ in Fig. 3a). Such behaviour resembles a transistor like functionality. With the initial value $I_{\text {SetPoint }}$ of $0.05 \mathrm{nA}$ at $E_{\mathrm{S}}=-0.80 \mathrm{~V}$, the tunnelling current increases to $\sim 0.65 \mathrm{nA}$ at the peak position, which corresponds to an on/off
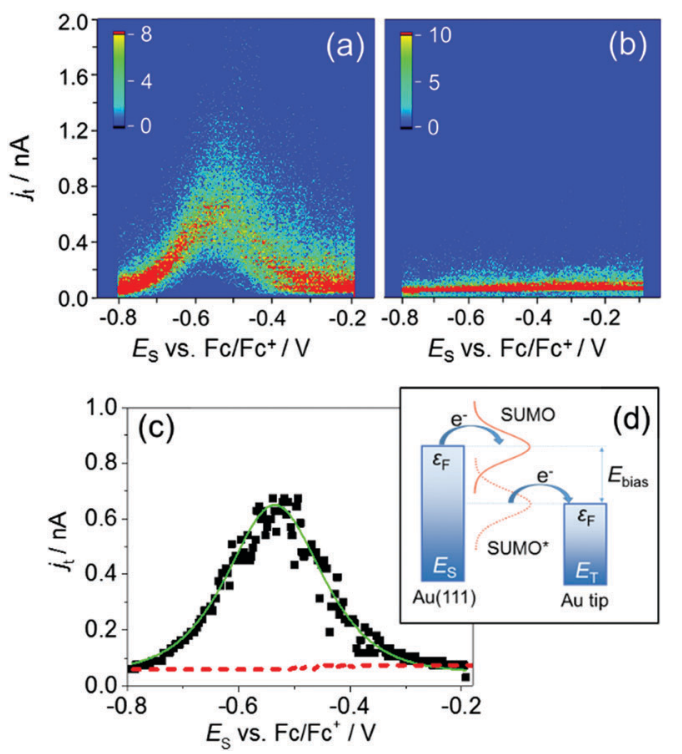

Fig. 4 2D occurrence histograms constructed from (a) 200 and (b) 100 constant bias current-voltage traces for SAM on Au(111): (a) PTM-C8-SH radical and (b) $\alpha \mathrm{H}-\mathrm{PTM}-\mathrm{C} 8-\mathrm{SH}$. Set-point current $/_{\text {SetPoint }}=50 \mathrm{pA}, E_{\text {bias }}=0.1 \mathrm{~V}$ (constant), starting $E_{\mathrm{S}}=-0.81 \mathrm{~V}$ and $E_{\mathrm{T}}=-0.71 \mathrm{~V}$. No data selection was applied. (c) Master current-voltage curves obtained from the data in panels (a) and (b): for the radical (squares) and $\alpha \mathrm{H}$-PTM (dashed line) forms. The solid green line represents fitting using the KU model (equations and fitting parameters are given in the ESI $\dagger$ ). (d) Schematic energy level diagram of a two-step ET process mediated by a redox-active radical molecule. ratio of $\sim 13$. The bell-shaped STS curves are typical for redoxmediated electron tunnelling (RMET) processes, which have been reported for a variety of redox-active molecules placed between the conductive substrate and the STM tip, ${ }^{2-24,26-32}$ as well as for single molecule junctions. ${ }^{11,47}$ However, it is the first time that a stable neutral free-radical is shown to mediate the tunnelling current enhancement under electrochemical conditions.

Similar to previous studies, the RMET phenomenon is rationalized by using the Kuznetsov-Ulstrup (KU) model, ${ }^{28}$ i.e., a two-step electron transfer with a partial vibrational relaxation. When the sample and the tip potentials are located before or after the redox peak, the Fermi levels of the two electrodes are lower and higher than the single-unoccupied molecular orbital (SUMO) of the PTM radical (Fig. 4d). In the first electron transfer step, the electron transfers from the electrode with a higher Fermi level to the SUMO. Afterwards, the energy of the now occupied SUMO* decreases due to vibrational relaxation. However, the second electron transfer occurs before a complete vibrational relaxation and before the energy of the molecular level passes the Fermi level of the second electrode. These electron-transfer steps are repeated causing a multitude of electrons to be transferred between the tip and the sample through this pathway, which is additional to the direct (off-resonant) tip-substrate current. Thus, the tunnelling current through the junction can be modulated by tuning the potential of both electrodes around the equilibrium potential of the $\mathrm{PTM}^{\bullet} / \mathrm{PTM}^{-}$redox pair.

By fitting the experimental data (Fig. 4c) using the KU model the following model parameter values were obtained: total (inner- + outer-sphere) reorganization energy $\lambda_{\mathrm{T}}=0.39 \mathrm{eV}$, fractions of overpotential and bias voltage at the redox site $\xi=0.57$ and $\gamma=0.57$, respectively (see the ESI $\dagger$ ). These values allow us to evaluate the efficiency of the PTM radical as a mediator compared to those reported in the literature. It should be kept in mind that the direct comparison of the fitted $\mathrm{KU}$ parameters between different redox systems is not straightforward since not all mediators are measured at the same fixed junction geometry in the STS experiments. Practically, this is hardly possible in an STM configuration and it would require, for example, systematic measurements of a series of nanodevices (with different mediators) with fixed electrode separation and molecular assembly (see discussion in ref. 25). Instead, the STS experiments are typically carried out at fixed starting $E_{\text {bias }}$ and $I_{\text {SetPoint }}$ (or tunnelling conductance $I_{\text {SetPoint }} / E_{\text {bias }}$ ) which, via the STM feedback mechanism, is expected to result in a reproducible junction geometry for the same "gap composition". Indeed, the established electrode separation will depend on the tunnelling efficiency through the gap in the absence of RMET (when the potentials of both electrodes are far from the equilibrium potential). Thus, diverse results attributed to a different gap geometry were obtained even for the same mediator in different starting redox states. ${ }^{23}$

Judging solely on the basis of the on/off ratio, the most effective mediators studied appear to be the Os bisterpyridine complex $\left(I_{\text {max }} / I_{\text {SetPoint }} \approx 50\right) .{ }^{22}$ This result was obtained in an ionic liquid using $E_{\text {bias }}=0.7 \mathrm{~V}$ and $I_{\text {SetPoint }}=0.05 \mathrm{nA}$, which is also the lowest tunnelling conductance used in EC-STS experiments. 
We cannot safely name this system as the most efficient mediator, as the on/off ratio tends to increase at lower tunnelling conductances. ${ }^{30}$ Formally, $I_{\text {max }} / I_{\text {SetPoint }} \approx 50$ was also obtained for viologens in one starting configuration $\left(E_{\mathrm{bias}}=0.05 \mathrm{~V}\right.$, $\left.I_{\text {SetPoint }}=0.1 \mathrm{nA}\right),{ }^{23}$ but the off-resonance current was asymmetric indicating the contribution of non-RMET enhancement. Among the results obtained at spontaneously established standard settings for STS experiments, $E_{\text {bias }}=0.1 \mathrm{~V}$ and $I_{\text {SetPoint }}=0.1 \mathrm{nA}$, the highest on/off ratios were obtained for perylene bisimide ${ }^{24}$ $\left(I_{\text {max }} / I_{\text {SetPoint }} \approx 35\right)$ and $\mathrm{Fc}(\mathrm{CO})\left(\mathrm{CH}_{2}\right)_{7} \mathrm{SH}^{29}\left(I_{\text {max }} / I_{\text {SetPoint }} \approx 12\right)$. To the best of our knowledge, a ratio $I_{\max } / I_{\text {SetPoint }}>10$ was not obtained for other mediators in either symmetric (covalently bonded to both contacts) ${ }^{11,47}$ or asymmetric (with a tunnelling gap) ${ }^{25,29,30,39}$ metal-molecule-metal junctions.

From the point of view of the KU model, an effective RMET mediator is characterized by a low total reorganization energy $(\lambda)$, which depends on tunnelling resistance and therefore on electrode separation. ${ }^{30}$ For example, in systems where $\lambda$ was estimated from the fitting of the STS results, the lowest values obtained were approximately $0.2 \mathrm{eV}$ for mediators such as perylene bisimide ${ }^{19}$ and $\lambda \approx 0.35-0.4 \mathrm{eV}$ for mediators such as the herein reported PTM and $\mathrm{Fc}(\mathrm{CO})\left(\mathrm{CH}_{2}\right)_{7} \mathrm{SH}^{29}$ Interestingly, perylene bisimide and Os bisterpyridine complexes are bulky structures, which is expected to decrease the outer-sphere reorganization energy associated with the surrounding solvent, leading to a higher $I_{\max } / I_{\text {SetPoint }}$.

To get further information about the system presented in this work, the PTM inner-sphere reorganization energy $\left(\lambda_{\mathrm{i}}\right)$ has been calculated using Density Functional Theory by finite energy differences ${ }^{48}$ giving a $\lambda_{\mathrm{i}}$ of $0.08 \mathrm{eV}$ (see the ESI $\dagger$ for further details). This value is comparable to $\lambda_{\mathrm{i}}$ calculated for perylene bisimide $(0.10 \mathrm{eV}$, see the $\mathrm{ESI} \dagger)$, but significantly higher than $\lambda_{\mathrm{i}}$ for ferrocene $(\sim 0.005 \mathrm{eV}$, as estimated from the spectroscopic data $\left.{ }^{49}\right)$. Nevertheless, with the on/off ratio 13 and estimated $\lambda=0.39 \mathrm{eV}$, the PTM radical is one of the best reported redox mediators in the RMET mechanism. As expected, the outer-sphere reorganization energy (estimated from the difference between the calculated $\lambda_{\mathrm{i}}$ and the experimental $\lambda_{\mathrm{T}}$ ) has a higher influence on the results.

In summary, in this work, we have prepared an organic radical based SAM on $\mathrm{Au}(111)$ exhibiting a reversible and stable redox signal in ionic liquids. Electrochemical scanning tunneling spectroscopy experiments demonstrated current enhancement due to the redox-mediated electron tunnelling mechanism. In comparison with other redox mediators studied to date, the PTM radical is among the most efficient ones, which indicates its potential applicability as an active electronic component in nanoelectronic devices.

We acknowledge the financial support from the EU projects ACMOL (FET Young Explorers, GA no. 618082), ERC StG 2012306826 e-GAMES, ITN iSwitch (GA no. 642196), COST Action TD1002, the Swiss National Science Foundation (Grant No. 200020144471), the Networking Research Center of Bioengineering, Biomaterials and Nanomedicine (CIBER-BBN), the DGI (Spain) with project BE-WELL CTQ2013-40480-R, the Generalitat de Catalunya with project 2014-SGR-17, and the Severo Ochoa program. N. C acknowledges the RyC program. C. F. is enrolled in the Materials Science PhD program of UAB. We acknowledge support of the publication fee by the CSIC Open Access Publication Support Initiative through its Unit of Information Resources for Research (URICI).

\section{Notes and references}

1 L. Sun, Y. A. Diaz-Fernandez, T. A. Gschneidtner, F. Westerlund, S. Lara-Avila and K. Moth-Poulsen, Chem. Soc. Rev., 2014, 43, 7378.

2 S. Guo, J. M. Artés and I. Díez-Pérez, Electrochim. Acta, 2013, 110, 741.

3 Nat. Nanotechnol., 2013, 8, 377.

4 C. A. Nijhuis, W. F. Reus and G. M. Whitesides, J. Am. Chem. Soc., 2010, 132, 18386.

5 M. Baghernejad, X. Zhao, K. Baruël Ørnsø, M. Füeg, P. Moreno-García, A. V. Rudnev, V. Kaliginedi, S. Vesztergom, C. Huang, W. Hong, P. Broekmann, T. Wandlowski, K. S. Thygesen and M. R. Bryce, J. Am. Chem. Soc., 2014, 136, 17922.

6 N. Darwish, I. Díez-Pérez, P. Da Silva, N. Tao, J. J. Gooding and M. N. Paddon-Row, Angew. Chem., Int. Ed., 2012, 51, 3203.

7 X. Xiao, L. A. Nagahara, A. M. Rawlett and N. Tao, J. Am. Chem. Soc., 2005, 127, 9235.

8 T. Avellini, H. Li, A. Coskun, G. Barin, A. Trabolsi, A. N. Basuray, S. K. Dey, A. Credi, S. Silvi, J. F. Stoddart and M. Venturi, Angew. Chem., Int. Ed., 2012, 51, 1611.

9 V. Kolivoska, M. Mohos, I. V. Pobelov, S. Rohrbach, K. Yoshida, W. J. Hong, Y. C. Fu, P. Moreno-Garcia, G. Meszaros, P. Broekmann, M. Hromadova, R. Sokolova, M. Valasek and T. Wandlowski, Chem. Commun., 2014, 50, 11757.

10 V. Kaliginedi, H. Ozawa, A. Kuzume, S. Maharajan, I. V. Pobelov, N. H. Kwon, M. Mohos, P. Broekmann, K. M. Fromm, M.-A. Haga and T. Wandlowski, Nanoscale, 2015, 7, 17685.

11 H. M. Osorio, S. Catarelli, P. Cea, J. B. G. Gluyas, F. Hartl, S. J. Higgins, E. Leary, P. J. Low, S. Martín, R. J. Nichols, J. Tory, J. Ulstrup, A. Vezzoli, D. C. Milan and Q. Zeng, J. Am. Chem. Soc., 2015, 137, 14319.

12 C. Huang, A. V. Rudnev, W. Hong and T. Wandlowski, Chem. Soc. Rev., 2015, 44, 889.

13 M. Mas-Torrent, N. Crivillers, C. Rovira and J. Veciana, Chem. Rev., 2012, 112, 2506.

14 N. Crivillers, M. Mas-Torrent, J. Vidal-Gancedo, J. Veciana and C. Rovira, J. Am. Chem. Soc., 2008, 130, 5499.

15 C. Simão, M. Mas-Torrent, N. Crivillers, V. Lloveras, J. M. Artés, P. Gorostiza, J. Veciana and C. Rovira, Nat. Chem., 2011, 3, 359.

16 C. Simão, M. Mas-Torrent, J. Veciana and C. Rovira, Nano Lett., 2011, 11, 4382.

17 N. Crivillers, M. Paradinas, M. Mas-Torrent, S. T. Bromley, C. Rovira, C. Ocal and J. Veciana, Chem. Commun., 2011, 47, 4664.

18 L. Yuan, C. Franco, N. Crivillers, M. Mas-Torrent, L. Cao, C. S. S. Sangeeth, C. Rovira, J. Veciana and C. A. Nijhuis, Nat. Commun., 2016, 7, 12066. 
19 N. Crivillers, C. Munuera, M. Mas-Torrent, C. Simão, S. T. Bromley, C. Ocal, C. Rovira and J. Veciana, Adv. Mater., 2009, 21, 1177.

20 R. Frisenda, R. Gaudenzi, C. Franco, M. Mas-Torrent, C. Rovira, J. Veciana, I. Alcon, S. T. Bromley, E. Burzurí and H. S. J. van der Zant, Nano Lett., 2015, 15, 3109.

21 J. Guasch, X. Fontrodona, I. Ratera, C. Rovira and J. Veciana, Acta Crystallogr., Sect. C: Cryst. Struct. Commun., 2013, 69, 255.

22 T. Albrecht, K. Moth-Poulsen, J. B. Christensen, J. Hjelm, T. Bjørnholm and J. Ulstrup, J. Am. Chem. Soc., 2006, 128, 6574.

23 I. V. Pobelov, Z. H. Li and T. Wandlowski, J. Am. Chem. Soc., 2008, 130, 16045.

24 C. Li, A. Mishchenko, Z. Li, I. Pobelov, T. Wandlowski, X. Q. Li, F. Würthner, A. Bagrets and F. Evers, J. Phys.: Condens. Matter, 2008, 20, 374122.

25 A. Alessandrini and P. Facci, Eur. Polym. J., 2016, 83, 450.

26 I. Díez-Pérez, Z. Li, S. Guo, C. Madden, H. Huang, Y. Che, X. Yang, L. Zang and N. Tao, ACS Nano, 2012, 6, 7044.

27 A. Mishchenko, M. Abdualla, A. Rudnev, Y. Fu, A. R. Pike and T. Wandlowski, Chem. Commun., 2011, 47, 9807.

28 J. Zhang, A. M. Kuznetsov, I. G. Medvedev, Q. Chi, T. Albrecht, P. S. Jensen and J. Ulstrup, Chem. Rev., 2008, 108, 2737.

29 A. V. Rudnev, I. V. Pobelov and T. Wandlowski, J. Electroanal. Chem., 2011, 660, 302.

30 Z. Li, Y. Liu, S. F. L. Mertens, I. V. Pobelov and T. Wandlowski, J. Am. Chem. Soc., 2010, 132, 8187.

31 P. Salvatore, A. Glargaard Hansen, K. Moth-Poulsen, T. Bjornholm, R. John Nichols and J. Ulstrup, Phys. Chem. Chem. Phys., 2011, 13, 14394.

32 J. M. Artés, I. Díez-Pérez and P. Gorostiza, Nano Lett., 2012, 12, 2679.

33 P. Petrangolini, A. Alessandrini and P. Facci, J. Phys. Chem. C, 2013, 117, 17451.
34 A. Alessandrini, S. Corni and P. Facci, Phys. Chem. Chem. Phys., 2006, 8, 4383.

35 N. J. Tao, Phys. Rev. Lett., 1996, 76, 4066.

36 X. Li, J. Hihath, F. Chen, T. Masuda, L. Zang and N. Tao, J. Am. Chem. Soc., 2007, 129, 11535.

37 T. Albrecht, K. Moth-Poulsen, J. B. Christensen, A. Guckian, T. Bjornholm, J. G. Vos and J. Ulstrup, Faraday Discuss., 2006, 131, 265.

38 T. Albrecht, A. Guckian, J. Ulstrup and J. G. Vos, IEEE Trans. Nanotechnol., 2005, 4, 430.

39 A. Alessandrini, M. Salerno, S. Frabboni and P. Facci, Appl. Phys. Lett., 2005, 86, 133902.

40 R. J. Nichols and S. J. Higgins, Electrocatalysis, Wiley-VCH Verlag GmbH \& Co. KGaA, 2013, p. 99.

41 J. M. Artés, M. López-Martínez, A. Giraudet, I. Díez-Pérez, F. Sanz and P. Gorostiza, J. Am. Chem. Soc., 2012, 134, 20218.

42 M. Gnahm and D. M. Kolb, J. Electroanal. Chem., 2011, 651, 250.

43 M. F. Suárez-Herrera, M. Costa-Figueiredo and J. M. Feliu, Langmuir, 2012, 28, 5286.

44 D. R. MacFarlane, J. M. Pringle, K. M. Johansson, S. A. Forsyth and M. Forsyth, Chem. Commun., 2006, 1905.

45 J. Veciana, J. Riera, J. Castañer and N. Ferrer, J. Organomet. Chem., 1985, 297, 131.

46 S. F. L. Mertens, G. Meszaros and T. Wandlowski, Phys. Chem. Chem. Phys., 2010, 12, 5417.

47 N. J. Kay, S. J. Higgins, J. O. Jeppesen, E. Leary, J. Lycoops, J. Ulstrup and R. J. Nichols, J. Am. Chem. Soc., 2012, 134, 16817.

48 S. F. Nelsen, S. C. Blackstock and Y. Kim, J. Am. Chem. Soc., 1987, 109, 677.

49 A. S. Baranski, K. Winkler and W. R. Fawcett, J. Electroanal. Chem. Interfacial Electrochem., 1991, 313, 367. 